Conclusions JAMs are differentially expressed in early and late SSc skin. Early SSc sera affect the expression and subcellular localisation of JAMs in dermal MVECs. In SSc, JAMs may participate in early inflammatory process, EC activation and impaired angiogenesis.

\title{
A65 DIFFERENTIAL EXPRESSION OF JUNCTIONAL ADHESION MOLECULES IN SYSTEMIC SCLEROSIS (SSC) SKIN
}

S Guiducci, ${ }^{1}$ M Manetti, ${ }^{1}$ A A F Milia, ${ }^{1}$ E Romano, ${ }^{1}$ C Ceccarelli, ${ }^{1}$ L Ibba-Manneschi, ${ }^{2}$ M Matucci-Cerinic ${ }^{1}{ }^{1}$ Biomedicine, Division of Rheumatology, University of Florence, Florence, Italy: ${ }^{2}$ Anatomy, Histology and Forensic Medicine, University of Florence, Florence, Italy

\subsection{6/ard.2010.149104.9}

Objective Junctional adhesion molecule (JAM)-A and JAM-C regulate leucocyte-endothelial cell (EC) interactions. JAMs also interact at inter-EC junctions regulating vascular permeability and are implicated in pathophysiological processes involving leucocyte transmigration, tight junction assembly and angiogenesis. SSc is characterised by early perivascular inflammatory infiltrates, EC damage and defective angiogenesis. We investigated the expression of JAMs in SSc skin and dermal microvascular ECs (MVECs) challenged with SSc sera. Methods Skin biopsies were obtained from the involved skin of 16 SSc patients (10 early, 6 late phase) and from 10 controls. Skin sections were stained with primary antibodies (Abs) anti-human JAM-A or JAM-C followed by fluorochromeconjugated secondary Abs. The lymphatic EC specific marker podoplanin (D2-40) was used to differentiate blood (D2-40-) and lymphatic (D2-40+) vessels. Human dermal MVECs were stimulated with VEGF or early SSc $(n=5)$ and control $(n=4)$ sera for 1, 6, $24 \mathrm{~h}$. MVECs were double-immunolabeled for JAM-C and the human tight junction protein zonula occludens-1 (ZO-1), or for JAM-A and the pro-angiogenic $\alpha \mathrm{V} \beta 3$ integrin. Immunostained tissue sections and cells were examined by confocal laser scanning microscopy. Densitometric analysis of staining intensity was performed using ImageJ software.

Results In control skin, constitutive expression of JAM-A was observed in blood and lymphatic ECs, fibroblasts and keratinocytes. In early SSc skin, JAM-A expression was increased in blood and lymphatic vessels and fibroblasts. Moreover, perivascular inflammatory cells showed strong JAM-A positivity. In late SSc, JAM-A expression was weaker than in controls. JAM-C was weakly expressed in controls. In early SSc, JAM-C expression was markedly observed in blood and lymphatic ECs, fibroblasts, and inflammatory cells. Instead, in late SSc JAM-C expression was similar to controls. Stimulation of MVECs with early SSc sera increased the cell surface expression of JAM-A and $\alpha V \beta 3$ integrin. JAM-C expression was found in MVEC cytoplasm at basal conditions and under stimulation with control sera. Upon early SSc sera challenging, JAM-C was rapidly recruited to tight junctions where it colocalised with ZO-1, with the maximun effect after $1 \mathrm{~h}$. Similar effects were observed after $1 \mathrm{~h}$ VEGF stimulation. 